Revista Destaques Acadêmicos, Lajeado, v. 9, n. 4, 2017. ISSN 2176-3070 DOI: http://dx.doi.org/10.22410/issn.2176-3070.v9i4a2017.1621 www.univates.br/revistas

\title{
ESTUDO DA FREQUÊNCIA DE RESSONÂNCIA DE UM SENSOR DE CORRENTES PARASITAS
}

\author{
Bruna Camacho' ${ }^{1}$, Sandro Gouveia ${ }^{2}$, Thomas Clarke ${ }^{3}$
}

Resumo: Ao realizar inspeção de descontinuidades da técnica de correntes parasitas, é muito importante definir a faixa de frequência de operação da bobina. A frequência de ressonância é o valor de limitação no qual a bobina para de ser um indutor e começa a funcionar como capacitor. Assim, a determinação da frequência de ressonância é primordial para o sucesso do uso da técnica de correntes parasitas. O objetivo deste trabalho é determinar experimentalmente a frequência de ressonância de uma bobina absoluta e analisar seus parâmetros e validar o resultado usando método de solução de elementos finitos (MEF). Para este fim, o pacote de software chamado COMSOL Multiphysics com módulo AC / DC foi utilizado.

Palavras-chave: Correntes Parasitas. COMSOL. Frequência de Ressonância.

\section{INTRODUÇÃO}

No desenvolvimento de sensores para inspeção de descontinuidades a partir de técnicas eletromagnéticas, como o ensaio não destrutivo por correntes parasitas (EC-NDT), alguns parâmetros são de suma importância para a obtenção de resultados confiáveis. Dentre tais parâmetros se destaca a faixa de frequência de operação da bobina.

A definição da faixa de frequência a ser trabalhada é diretamente dependente de dois fatores: a profundidade da descontinuidade em relação à superfície e o material a ser inspecionado. Existe, porém, um valor limite de frequência de operação no qual a bobina deixa de ser um indutor passando a trabalhar como um capacitor, tal valor é denominado frequência de ressonância (SHULL, 2002). Assim, determinar a frequência de ressonância é primordial para o sucesso do uso da técnica de correntes parasitas.

1 Engenheira Metalúrgica - LAMEF/Universidade Federal do Rio Grande do Sul.

2 Engenheiro Mecânico - LAMEF/Universidade Federal do Rio Grande do Sul.

3 Doutor, Professor - LAMEF/Universidade Federal do Rio Grande do Sul. 
As bobinas são comumente caracterizadas pelo parâmetro denominado impedância (Z), que consiste na relação entre a tensão (V) em Volts e a corrente (I) em Ampères e é medida em ohms conforme equação 1 abaixo.

$$
Z=\frac{V}{I}
$$

A impedância é uma medida complexa explicita por sua componente real, a resistência $(R)$, e por sua componente imaginária, a reatância $(X)$. A reatância ainda é composta pela reatância indutiva $\left(X_{L}\right)$, diretamente proporcional à frequência de excitação da tensão na bobina, e pela reatância capacitiva $\left(X_{c}\right)$, inversamente proporcional à frequência de excitação da tensão na bobina. Vide equações 2, 3 e 4; nas quais L é a indutância da bobina (em henry) e C é a capacitância do sistema (em farad).

$$
\begin{aligned}
& Z=\sqrt{\left(R^{2}+\left(X_{L}-X_{C}\right)^{2}\right.} \\
& X_{L}=2 \pi f L \\
& X c=\frac{1}{2 . \pi \cdot f . C}
\end{aligned}
$$

Um indutor ideal não apresenta efeitos resistivos nem capacitivos, sendo assim constituído somente de indutância. Porém, isso não é viável na prática e ele possui uma resistência em série no fio usado para enrolá-lo e uma capacitância distribuída entre os enrolamentos.

A impedância ainda está associada a uma amplitude e a um ângulo de fase. Assim, toda mudança de impedância é acompanhada de uma mudança na amplitude e na fase do sinal e a mudança na fase traz também informações sobre as condições do material (KEISER, 1979). Observa-se na equação 5 e na Figura 1 abaixo, o ângulo de fase que indica a defasagem da corrente, em relação à tensão. Em um capacitor ideal, o ângulo de fase será $-90^{\circ}$ e para um indutor ideal o ângulo de fase será $90^{\circ}$.

$$
\theta=\arctan \frac{X l-X c}{R}
$$


Figura 1-Representação da impedância no plano complexo.

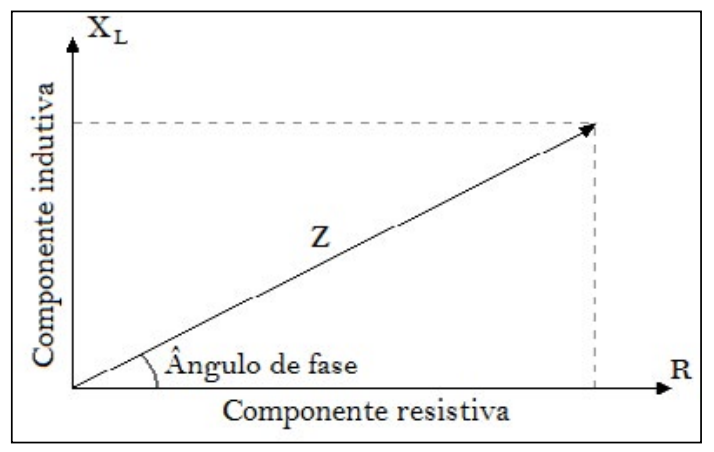

Fonte: dos autores

A ressonância ocorre a uma frequência de ressonância particular na qual as partes imaginárias da impedância, as reatâncias, se cancelam. Tal fenômeno ocorre quando a impedância entre a entrada e a saída do circuito da bobina é próxima de zero e, portanto, o ângulo de fase é zero. A equação 6 abaixo expressa a frequência de ressonância.

$$
f=\frac{1}{2 \cdot \pi \cdot \sqrt{L . C}}
$$

Abaixo da frequência de ressonância, o indutor se comporta como um indutor com poucas perdas. Na frequência de ressonância, ele comporta-se como um resistor e acima da frequência de ressonância comporta-se como um capacitor com uma reatância capacitiva finita decrescendo com o aumento da frequência. Dessa forma, tomando-se por base o valor da corrente, o ângulo de fase será positivo quando o arranjo for indutivo e será negativo quando o arranjo for capacitivo.

O presente trabalho tem como objetivo determinar experimentalmente a frequência de ressonância de uma bobina absoluta analisando seus parâmetros e validando os resultados através de simulação computacional pelo método de elementos finitos (MEF).

\section{DESENVOLVIMENTO}

Na realização da simulação por elementos finitos, foi utilizado o software COMSOL Multiphysics ${ }^{\circledR}$ no módulo AC/DC e na interface Magnetic and Electric Field (mef). Visando otimizar a memória do processador bem como reduzir o tempo de processamento, o modelo foi construído no ambiente " $2 \mathrm{D}$ axisymmetric".

A Figura 2 abaixo demonstra o modelo construído na simulação. As interações foram realizadas no domínio da frequência e alimentadas com tensão fixa de $0.500 \mathrm{~V}$. Os parâmetros de material associados a cada domínio descrito 
na Figura 2 estão listados na Tabela 1. A geometria do modelo foi construída no próprio ambiente do software seguindo os parâmetros descritos na Tabela 2. As modelagens foram realizadas em 91 pontos ao longo de uma faixa larga de frequência, que compreende de $1.000 .000 \mathrm{~Hz}$ a $10.000 .000 \mathrm{~Hz}$, a fim de visualizar a frequência de ressonância e o comportamento da bobina. Foram analisados os valores de impedância $(Z)$, resistência $(R)$, reatância $(X)$ e ângulo de fase $\left(^{\circ}\right)$.

Tabela 1 - Parâmetros do material no COMSOL

\begin{tabular}{c|c|c}
\hline $\begin{array}{c}\text { DOMINIO } \\
\text { (FIGURA 2) }\end{array}$ & $\begin{array}{c}\text { CONDUTIVIDADE } \\
\text { ELETRICA }\end{array}$ & $\begin{array}{c}\text { PERMEABILIDADE } \\
\text { RELATIVA }\end{array}$ \\
\hline (1) $\mathrm{Ar}$ & 0 & 1 \\
\hline (2) Cobre & $5.998 \mathrm{e} 7$ & 1 \\
\hline
\end{tabular}

Fonte: dos autores.

Figura 2 - Geometria da bobina no COMSOL

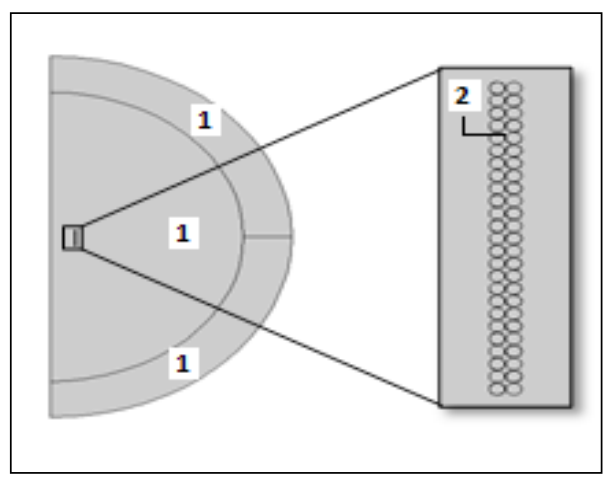

Fonte: dos autores.

Ainda, uma correta configuração da malha é fundamental a fim de minimizar a diferença entre a solução exata e a aproximada. Dessa forma, dois refinamentos de malha distintos foram aplicados nesse modelo, conforme se observa na Figura 3 abaixo. 
Figura 3 - Malha do modelo no COMSOL

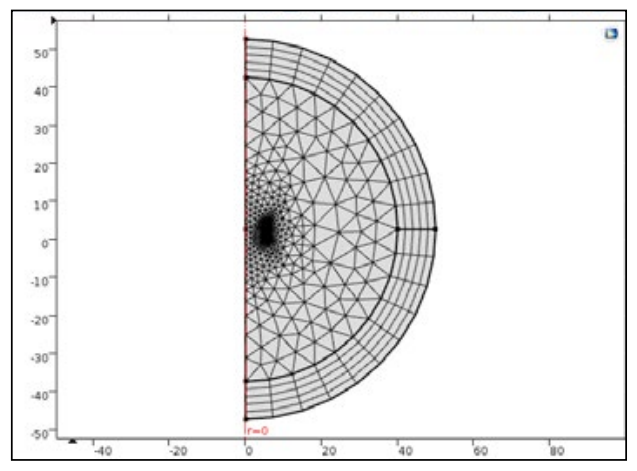

Fonte: dos autores.

O arranjo experimental é constituído de uma única bobina (bobina absoluta). Sondas compostas de uma bobina absoluta são amplamente utilizadas devido a sua versatilidade, podendo ser aplicadas para detecção de defeitos, caracterização de materiais e diagnóstico de espessura de revestimento. Quando se trata de caracterização de materiais, estas sondas são as mais recomendadas devido a sua grande sensibilidade à condutividade, permeabilidade e lift-off (LAI, 2005).

Os parâmetros da bobina estão descritos na Tabela 2 abaixo. A mesma foi construída com fio AWG35, classificado pela escala americana de fio ("American Wire Gauge") (FERREIRA, BOGGIO, 1973). Suas características estão listadas na Tabela 3. O interior do arranjo, vide Figura 4, foi confeccionado em material polimérico que, devido seu valor de permeabilidade magnética insignificante, não será considerado no estudo.

Tabela 2 - Parâmetros da Bobina Experimental.

\begin{tabular}{l|l}
\hline Diâmetro Fio & AWG 35-0.1426 [mm] \\
\hline Número de voltas & 50 \\
\hline Camadas em $X$ & 2 \\
\hline Camadas em $Y$ & 25 \\
\hline Diâmetro Interno & $10.1[\mathrm{~mm}]$ \\
\hline
\end{tabular}

Fonte: dos autores. 
Tabela 3 - Propriedades do fio AWG35

\begin{tabular}{c|c|c|c}
\hline AWG & Diâmetro [mm] & $\begin{array}{c}\text { Seção circular } \\
{\left[\mathbf{m m}^{2}\right]}\end{array}$ & $\begin{array}{c}\text { Resistência [ohms/ } \\
\mathbf{k m}]\end{array}$ \\
\hline 35 & 0,1426 & 0,0159 & 1,069 \\
\hline
\end{tabular}

Fonte: dos autores.

Figura 4 - Bobina experimental

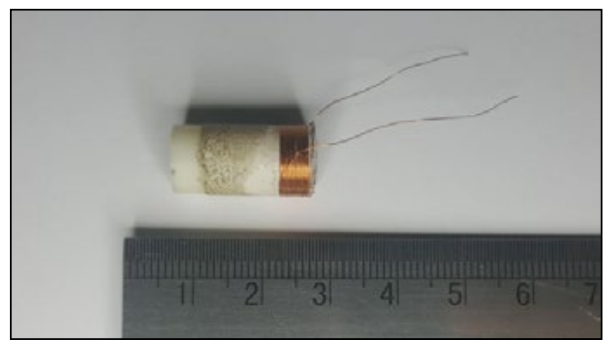

Fonte: dos autores.

A realização das medidas no arranjo experimental foi efetuada no equipamento medidor de impedância a uma tensão de alimentação de 0.500 Volts na faixa de frequência de $1.000 .000 \mathrm{~Hz}$ a $10.000 .000 \mathrm{~Hz}$. Foram analisados os valores de impedância $(Z)$, resistência $(R)$, reatância $(X)$ e ângulo de fase $\left(^{\circ}\right)$ em 801 pontos igualmente distribuídos pelo equipamento ao longo da faixa de frequência selecionada.

\section{RESULTADOS E DISCUSSÃO}

Nas Figuras 5 e 6 estão os gráficos referentes às medidas dos módulos da impedância $(Z)$, da resistência $(R)$, da capacitância $(X)$ e do ângulo de fase $\left(^{\circ}\right)$ obtidas através da simulação no software e da medição do arranjo experimental, respectivamente.

Através do modelo simulado constatou-se a ressonância no valor de frequência de $6.800 .000 \mathrm{~Hz}$, e através do arranjo experimental constatou-se a ressonância em $6.951 .250 \mathrm{~Hz}$. A precisão entre os valores de frequência de ressonância na simulação e no arranjo experimental é bastante satisfatória e a diferença entre os dois valores pode ser explicada a partir de fatores externos e alheios ao controle do operador, tais como oscilações de temperatura e vibrações do ambiente, e pelo fato de o número de medidas no arranjo experimental serem aproximadamente 10 vezes maior que o número de medidas simuladas. 
Figura 5 - Curvas obtidas através da simulação no COMSOL

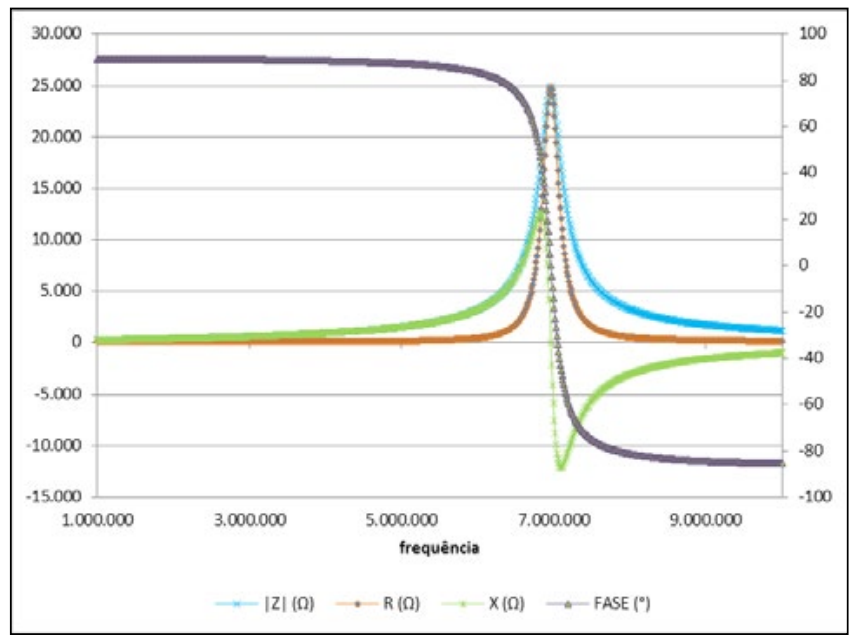

Fonte: dos autores.

Figura 6 - Curvas obtidas na medição do arranjo experimental

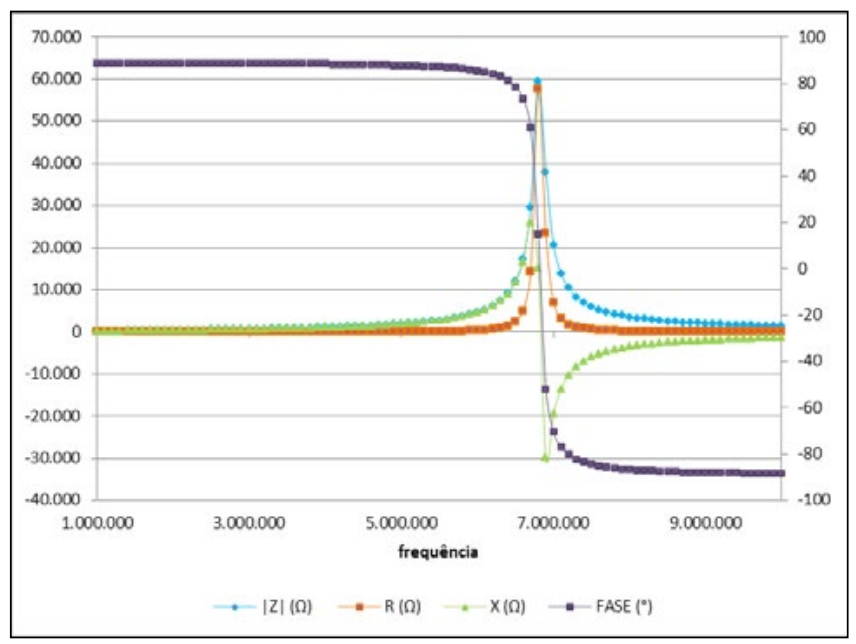

Fonte: dos autores.

Nas Figuras 7 e 8 estão os gráficos referentes às medidas da impedância $(Z)$ e do ângulo de fase $\left({ }^{\circ}\right)$ obtidas através da simulação no software e da medição do arranjo experimental, respectivamente. 
Figura 7 - Curvas de impedância $(Z)$ e ângulo de fase $\left({ }^{\circ}\right)$ obtidas através da simulação no COMSOL

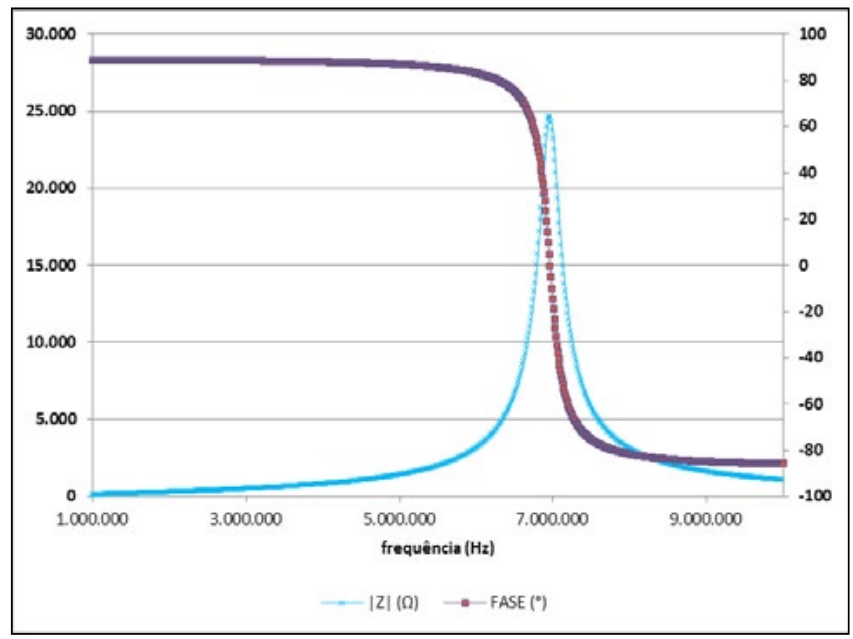

Fonte: dos autores.

Figura 8 - Curvas de impedância $(Z)$ e ângulo de fase $\left(^{\circ}\right)$ obtidas na medição do arranjo experimental

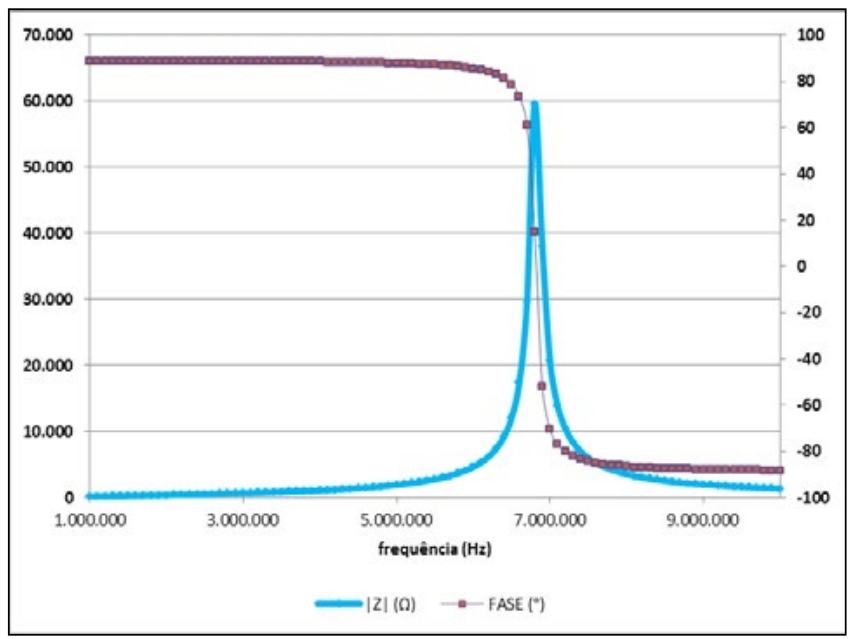

Fonte: dos autores.

Observa-se que na medida em que a frequência tende a zero anterior à frequência de ressonância observada nos picos das curvas de impedância em ambos os gráficos, o ângulo de fase tende a ser negativo, ou seja, a bobina tem comportamento capacitivo. Porém, quando a frequência tende a infinito posteriormente à frequência de ressonância observada nos picos das curvas de 
impedância em ambos os gráficos, o ângulo de fase tende a ser positivo, ou seja, a bobina tem comportamento indutivo.

Finalmente, quando a frequência é igual a de ressonância, o ângulo de fase se comporta de modo a representar a diferença de fase nula e, neste caso, a bobina apresenta comportamento puramente resistivo.

Nas Figuras 9 e 10 estão os gráficos referentes às medidas da impedância $(Z)$ e da reatância $(X)$ obtidas através da simulação no software e da medição do arranjo experimental, respectivamente.

Um indutor se comporta de forma inversa à forma de comportamento de um capacitor. Enquanto a corrente alternada, corrente aplicada no caso deste estudo, passa livremente pelo capacitor, no indutor a corrente contínua passa sem resistência enquanto a corrente alternada encontra a resistência gerada pelo campo magnético criado pelo indutor (SHULL, 2002). Essa resistência é a reatância indutiva e varia proporcionalmente de acordo com a frequência da corrente, ou seja, quanto maior a frequência da corrente, maior a reatância indutiva. Sendo assim observa-se o comportamento das curvas de reatância em ambos os gráficos: anterior à frequência de ressonância a bobina se comporta como um indutor e seu valor de reatância indutiva cresce proporcionalmente à frequência aplicada, já após a frequência de ressonância a bobina assume comportamento capacitivo e seu valor de reatância tende a se aproximar do valor nulo à medida que aumenta a frequência.

Na faixa de frequência próxima à ressonância observam-se os maiores valores de reatância e, consequentemente, os picos nas curvas de impedância devido à oposição à passagem de corrente quando submetido à tensão. Isso ocorre poque na região a resistência a passagem de corrente é máxima, seja medida e constatada no valor de reatância indutiva, de reatância capacitiva ou no valor nulo de reatância que ocorre na frequência de ressonância na qual a bobina se mostra puramente resistiva, sendo a impedância caracterizada pela medida da resistência $(R)$. 
Figura 9 - Curvas de impedância $(Z)$ e reatância $(X)$ obtidas através da simulação no COMSOL

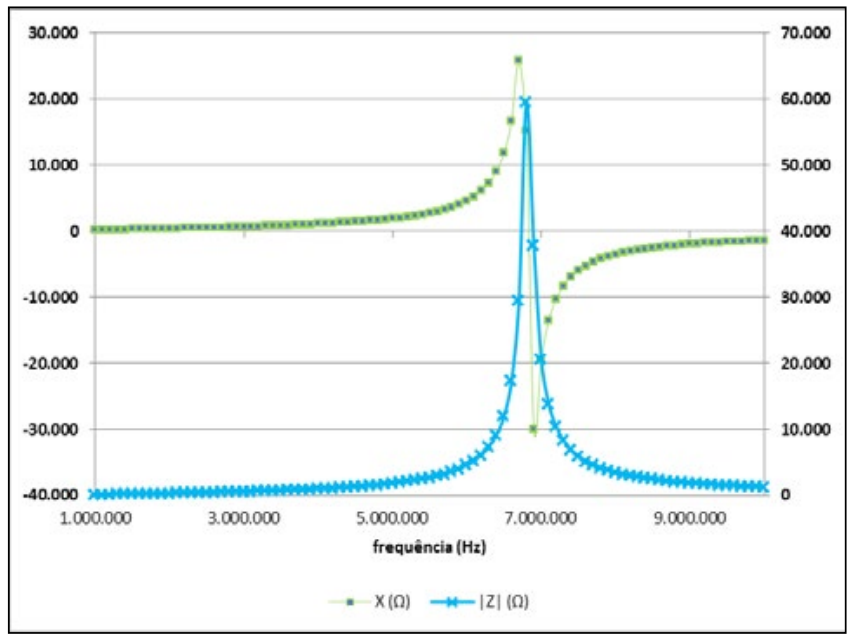

Fonte: dos autores.

Figura 10 - Curvas de impedância $(Z)$ e reatância $(X)$ obtidas na medição do arranjo experimental

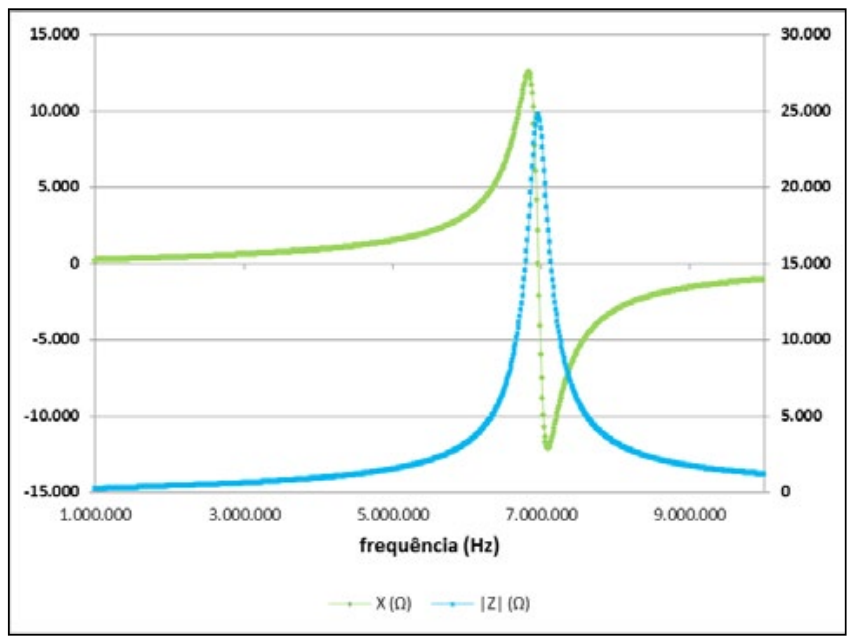

Fonte: dos autores.

\section{CONCLUSÕES}

O objetivo do trabalho foi estimar o valor de frequência no qual ocorre a ressonância em uma bobina absoluta utilizando o método de elementos 
finitos de solução e a comparação com as medidas experimentais. A precisão entre os resultados obtidos através da modelagem no COMSOL e do arranjo experimental foi bastante satisfatória validando, portanto, tal metodologia em bobinas absolutas. A geometria e o tamanho da malha possuem influência determinante sobre os resultados finais e induzem a mudanças nos valores obtidos através da simulação no COMSOL.

O valores obtidos de resistência, reatência, impedância e ângulo de fase, bem como seu comportamento ao longo da faixa de frequência estudada, são verossímeis com a literatura e com a análise analítica através das equações citdas no início do trabalho. Um trabalho futuro deve ser focada na exposição dos cálculos analíticos e no aprofundamento da comparação de seus resultados com os obtidos na simulação e no arranjo experimental.

O método de elementos finitos de solução pode ainda ser usado para simular a identificação de descontinuidades, tais como a presença de trincas superficiais e subsuperficiais e efeitos da ação corrosiva do meio, em materiais metálicos através da bobina. Trabalhos futuros serão focados nesse segmento de simulação e na comparação com resultados experimentais.

\section{REFERÊNCIAS}

FERREIRA, J. C. and BOGGIO, S. A. Tabela de fios esmaltados padrão americano AWG para referência aos construtores de transformadores. Monitor, vol. 4 pp. 46 -47, 1973.

KEISER, B. E. Principles of electromagnetic compatibility. Dedham, Massachussetts: Artech House, Inc., 1979.

LAI, Y. Eddy current displacement sensor with LTCC technology. PhD Thesis, Albert-Ludwigs Universität Freiburg im Breisgau, 2005

SHULL, P. Nondestructive Evaluation: Theory, Techniques and Applications. 1a edição. Altoona 2002. 